7. Reprod. Fertil. (1962) 4, 121-124

\title{
EXPERIMENTS ON INTERSPECIFIC SPERM PENETRATION THROUGH THE ZONA PELLUCIDA
}

\author{
Z. DICKMANN* \\ A.R.C. Unit of Reproductive Physiology and Biochemistry, Cambridge University
}

(Received 20th December 1961)

\begin{abstract}
Summary. Rat eggs were transferred to the oviducts of previously mated rabbits. The purpose of such transfers was to find out whether or not spermatozoa of one species can penetrate through the zona pellucida of another species. Of the 673 rat eggs that were examined, the perivitelline space of one contained a rabbit spermatozoon. Penetration in this single instance seemed to have occurred due to abnormalities in the spermatozoon.
\end{abstract}

\section{INTRODUCTION}

Few facts are available concerning the mechanism involved in sperm penetration through the zona pellucida. This mechanism was elucidated, in part, on the basis of observations made by Austin \& Bishop (1958) who postulated that the spermatozoon attaches itself to the zona in the region of its acrosome. Then, due to its movement, the spermatozoon becomes detached from its acrosome which exposes the perforatorium, an organelle at the anterior end of the sperm head. The perforatorium is believed to enable the spermatozoon to penetrate through the zona, possibly by means of an enzyme. The enzyme in question, referred to as zona lysin, has not, as yet, been isolated and, therefore, must be regarded as hypothetical.

In an attempt to further elucidate the mechanism of sperm penetration through the zona, the present series of experiments were designed to determine whether or not spermatozoa of one species (rabbit) can penetrate through the zona of another species (rat).

\section{MATERIALS AND METHODS}

Three series of experiments (Series 1, 2 and 3) were conducted, all based on the same design; however, in the two latter series the original design was altered in one or more of its details.

SERIES 1

The technique used throughout these experiments was to transfer rat eggs into the oviducts of previously mated rabbits. The egg donors were immature rats 29 to 34 days old, in which superovulation was induced by subcutaneous injection of 30 i.u. of pregnant mare's serum (Gestyl, Organon Laboratories) followed 52 to $56 \mathrm{hr}$ later by $20 \mathrm{i} . \mathrm{u}$. of chorionic gonadotrophin (Pregnyl,

* Present address: Animal Research Station, 307 Huntingdon Road, Cambridge. 
Organon Laboratories). One to $3 \mathrm{hr}$ after ovulation had occurred, the rats were sacrificed, their oviducts removed and the eggs recovered, always surrounded by cumulus. In different transfers, three types of media were used: (1) equal parts of rabbit plasma and physiological saline solution $(0.9 \% \mathrm{NaCl}) ;(2)$ Ringer's solution; (3) physiological saline solution. The eggs were kept in vitro, at room temperature, for 40 to $60 \mathrm{~min}$, and were then transferred into both oviducts of the recipient rabbit. Ten to $12 \mathrm{hr}$ prior to the transfer, the recipient rabbit had been mated to two fertile bucks in rapid succession. Thus, the eggs were transferred while ovulation in the recipient was in progress. (Rabbits normally ovulate 10 to $12 \mathrm{hr}$ post coitum.) Seven to $11 \mathrm{hr}$ after the transfer the recipient was sacrificed and her oviducts were removed and flushed in order to recover both the native and the transferred eggs. The rat eggs were then examined to see whether rabbit spermatozoa had penetrated through their zonae. The native rabbit eggs served as controls by which to ascertain that spermatozoa had reached the site of fertilization.

SERIES 2

Rat eggs were obtained as in Series 1 , but immediately after recovery they were denuded of their cumulus by placing them in a medium containing hyaluronidase (Hyalase, Benger Laboratories). Following denudation, the eggs were washed in a hyaluronidase-free medium, 'sandwiched' between two pieces of cumulus obtained from donor rabbits (the rabbit eggs not having been removed from these cumuli), and were then transferred to the oviducts of recipient rabbits. Denudation of the rat eggs was carried out because of the possibility that rabbit spermatozoa might not be able to penetrate through rat cumulus. The reason for embedding the eggs in rabbit cumulus was twofold: (1) the cumulus may aid in 'trapping' spermatozoa, and (2) the speed of egg transport through the oviduct may be altered in the absence of cumulus.

SERIES 3

In these experiments, the rat eggs were recovered, not at 1 to $3 \mathrm{hr}$, but at 7 to $10 \mathrm{hr}$ after ovulation, at which time they are still surrounded by cumulus. This modification was adopted with the possibility in mind that the zonae of slightly aged eggs might be less resistant to the penetration of foreign spermatozoa.

\section{RESULTS}

The proportions of rat and rabbit eggs recovered are summarized in Table 1. It was found that all the rabbit eggs, with the exception of four (one in Series 1 and three in Series 2) were penetrated by one or more spermatozoa, which indicated that rabbit spermatozoa were available for penetration of the transferred rat eggs.

Of the 673 rat eggs recovered, none contained spermatozoa in their zonae; however, the perivitelline space of one egg (in Series 2) contained a spermatozoon (Pl. 1, Fig. 1). This egg was rolled under the coverslip, a technique by which one can ascertain beyond any doubt whether a spermatozoon is inside 
PLATE 1
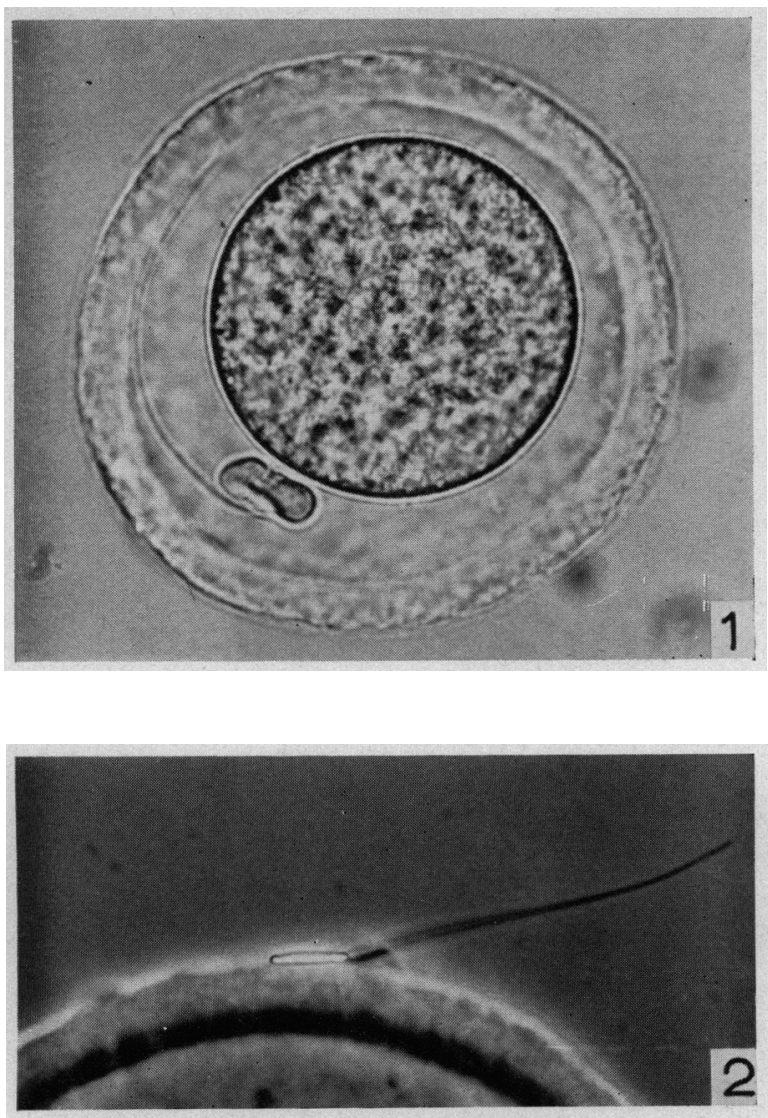

Fig. 1. Rat egg containing a rabbit spermatozoon in its perivitelline space. $\times 560$. FiG. 2. Rabbit spermatozoon attached to the zona pellucida of a rat egg. Phase-contrast. $\times 900$.

(Facing p. 122) 
the perivitelline space or merely on the surface of the zona. The sperm head, however, was not seen. It is possible that it was attached to the polar body in such a way that it escaped observation (see Pl. 1, Fig. 1). It might also have disintegrated in the fluid of the perivitelline space. The length of the sperm tail, as measured on the photograph, was $50 \%$ greater than the normal. Such an exaggerated length undoubtedly indicates an abnormality.

In seventy-three of the rat eggs recovered, either one or two rabbit spermatozoa were found attached to each of their zonae, as is shown in Pl. 1, Fig. 2.

TABLE 1

PROPORTION OF RAT AND RABBIT EGGS RECOVERED

\begin{tabular}{c|cc|c|cc}
\hline Series & $\begin{array}{c}\text { Proportion of trans. } \\
\text { ferred rat eggs } \\
\text { recovered }\end{array}$ & $\begin{array}{c}\text { No. rabbit ovi- } \\
\text { ducts to which eggs } \\
\text { transferred }\end{array}$ & $\begin{array}{c}\text { Proportion of rabbit } \\
\text { eggs recovered }\end{array}$ \\
\hline 1 & $\frac{394}{468}$ & $(84 \%)$ & 22 & $\frac{96}{110}$ & $(87 \%)$ \\
\hline 2 & $\frac{136}{168}$ & $(81 \%)$ & 10 & $\frac{86}{91}$ & $(95 \%)$ \\
\hline 3 & $\frac{143}{174}$ & $(82 \%)$ & 10 & $\frac{46}{48}$ & $(96 \%)$ \\
\hline Total & $\frac{673}{810}$ & $(83 \%)$ & 42 & & \\
\hline
\end{tabular}

* Includes the rabbit eggs which were transferred in the cumulus.

\section{DISGUSSION}

The present experiments were aimed at determining whether spermatozoa can penetrate through the zona of an egg of an unrelated species. The results indicate that, under the conditions of the experiments, the rabbit spermatozoon is normally unable to penetrate through the zona of the rat egg. However, it is of interest that on a single occasion sperm penetration did take place. It is tempting to speculate that the rabbit spermatozoon which penetrated through the zona of the rat egg was able to do so because it was an abnormal spermatozoon. In fact, it bore a visible abnormality - the length of its tail, which was $50 \%$ greater than the normal. An abnormal zona of the rat egg in question could also have affected penetration, but there were no visible indications of abnormality.

Why is the rabbit spermatozoon unable to penetrate through the zona of the rat egg? Many explanations can undoubtedly be offered, but at present only four will be outlined.

(I) The morphology of the spermatozoon is likely to have a bearing on its ability to penetrate through the zona. Rat and rabbit spermatozoa differ markedly in several morphological aspects: the head of the rat spermatozoon is hook-shaped, whereas that of the rabbit is oval; the length of the head is A* 
$11 \cdot 7 \mu$ versus $8.4 \mu$, midpiece is $67 \mu$ versus $8 \mu$, and mainpiece is $110 \mu$ versus $38 \mu$ in rat and rabbit, respectively (measurements from Bishop \& Walton, 1960).

(2) Earlier in this paper it was mentioned that the spermatozoon may depend on an enzyme, referred to as zona lysin, for its penetration through the zona. It is possible that the zona lysin of the rabbit's spermatozoon differs chemically from that of the rat and therefore penetration is prevented.

(3) The rabbit oviduct may be an unfavourable environment for the rat egg, causing the zona to undergo a reaction which would render it impenetrable.

(4) Acrosome detachment, presumably a prerequisite for sperm penetration through the zona, can probably proceed only if the head of the spermatozoon initially attaches itself to the zona solely by its acrosome. If the full length of the sperm head was to attach to the zona, which occurred when rabbit spermatozoa attached to rat zonae (see Pl. 1, Fig. 2), acrosome detachment and therefore sperm penetration through the zona may be prevented.

\section{ACKNOWLEDGMENTS}

The author wishes to thank Drs C. E. Adams, P. J. Dziuk and T. R. R. Mann and Mr L. E. A. Rowson for reading the manuscript.

Financial support from the Population Council is gratefully acknowledged.

\section{REFERENCES}

Austin, G. R. \& Bishop, M. W. H. (1958) Role of the rodent acrosome and perforatorium in fertilization. Proc. roy. Soc. B, 149, 241.

Bishop, M. W. H. \& WALron, A. (1960) Spermatogenesis and the structure of mammalian spermatozoa. Marshall's Physiology of Reproduction, 3rd edn., vol. 1, pt. 2, p. 1. Ed. A. S. Parkes. Longmans, Green, London. 